\title{
Rice CASP1 regulates suberin deposition in small lateral roots and plays crucial role in metal homeostasis in plant
}

\author{
Xianfeng Yang ${ }^{1}$, Huifang Xie ${ }^{1}$, Qunqing Weng ${ }^{1}$, Kangjing Liang ${ }^{1}$, Xiujuan Zheng ${ }^{1}$, Yuchun \\ Guo $^{1}$, and Xinli Sun ${ }^{1}$ \\ ${ }^{1}$ Fujian Agriculture and Forestry University
}

June 22, 2020

\begin{abstract}
Arabidopsis Casparian strip membrane domain proteins (CASPs) form a transmembrane scaffold to recruit lignin biosynthetic enzymes for Casparian strip (CS) formation. Compared with Arabidopsis, rice root is more complex with a CS of the exodermis and sclerenchyma and a CS that does not block propidium iodide entry into the stele. Rice CASP1 is highly similar to AtCASPs, but it is not required for CS formation. Its mutation results in early leaf senescence and fewer tillers and does not change the CS structure and permeability. OsCASP1 is mainly located in the nuclear membrane. Its expression is concentrated in the root stele and at small lateral root tips and can be induced by salt stress. OsCASP1 mutation causes ectopic suberin deposition in small lateral roots and ion imbalances in the plant. Homeostatic disorder induces nutrient recycling and accelerate leaf senescence. To our knowledge, OsCASP1 is the first CASP to be described in the nuclear membrane; it modulates suberin deposition and does not involve CS formation, representing a novel regulatory mode of CASPs.
\end{abstract}

Rice CASP1 regulates suberin deposition in small lateral roots and plays crucial role in metal homeostasis in plant

Running Head: OsCASP1 modulates suberin deposition

Xianfeng Yang*, Huifang Xie*, Qunqing Weng*, Kangjing Liang, Xiujuan Zheng, Yuchun Guo, Xinli Sun**

* These authors contributed equally to this work

** To whom correspondence should be addressed: xinlisun@hotmail.com

Key Laboratory of Ministry of Education for Genetics, Breeding and Multiple Utilization of Crops, Fujian Agriculture \& Forestry University, Fuzhou, 350002, China

College of Agriculture, Fujian Agriculture \& Forestry University, Fuzhou, 350002, China

Abstract: Arabidopsis Casparian strip membrane domain proteins (CASPs) form a transmembrane scaffold to recruit lignin biosynthetic enzymes for Casparian strip (CS) formation. Compared withArabidopsis , rice root is more complex with a CS of the exodermis and sclerenchyma and a CS that does not block propidium iodide entry into the stele. Rice CASP1 is highly similar to AtCASPs, but it is not required for CS formation. Its mutation results in early leaf senescence and fewer tillers and does not change the CS structure and permeability. OsCASP1 is mainly located in the nuclear membrane. Its expression is concentrated in the root stele and at small lateral root tips and can be induced by salt stress. OsCASP1 mutation causes ectopic suberin deposition in small lateral roots and ion imbalances in the plant. Homeostatic disorder induces nutrient recycling and accelerate leaf senescence. To our knowledge, OsCASP1 is the first CASP to be described in the nuclear membrane; it modulates suberin deposition and does not involve CS formation, representing a novel regulatory mode of CASPs. 
Key words: rice, CASP, Casparian strip, suberin deposition, leaf senescence

\section{Introduction}

Plant roots acquire nutrients from soil and transport them across all external cell layers into the central vasculature and then upward to the aerial parts. Water and nutrients move radially into the stele through a combination of three pathways. The first is the apoplastic pathway, where solutes diffuse in free spaces and cell walls of the epidermis and cortex, which can be completely blocked by Casparian strips (CS) (Barberon, 2017; Barberon \& Geldner, 2014; Doblas, Geldner, \& Barberon, 2017). The second is the symplastic pathway involving cell-to-cell transport via plasmodesmata, and the third is the coupled transcellular pathway, where polarized influx and efflux carriers transport solutes in a vectorial fashion (Barberon, 2017; Barberon \& Geldner, 2014; Doblas et al., 2017). The solutes obstructed by CS are transported into endodermal cells by relevant influx carriers and then move into the stele via efflux carriers and/or plasmodesmata. Suberin lamellae do not block apoplastic transport but rather limit transcellular transport of nutrients(Barberon, 2017; Doblas et al., 2017), which coat the entire endodermal cell surface between the plasma membrane and secondary cell wall and isolate the solute from carriers(Robbins II, Trontin, Duan, \& Dinneny, 2014).

CS formation is initiated at Casparian strip domain proteins (CASPs) at the Casparian strip membrane domain (CSD) in Arabidopsis . AtCASPs form a platform to localize lignin biosynthetic enzymes (Lee, Rubio, Alassimone, \& Geldner, 2013; Roppolo et al., 2011), and at least eleven other proteins modulate CS formation on the platform (Table S1). Suberin deposition is regulated by many genes and by different nutrient stresses, ABA and ethylene (Barberon et al., 2016). The mutants of CS formation, excluding sgn3, cause ectopic suberin deposition inArabidopsis (Barberon, 2017; Doblas et al., 2017) and usually alter the ion permeability and sensitivity to salt and drought stress (Table S1). Loss of integrity of the CS is sensed by small peptides (CIF1/2) from the stele into the cortex, which leads to enhanced suberin deposition and rebalanced water and mineral nutrient uptake (Nakayama et al., 2017; P. Wang et al., 2019).

There are 5 CASPs and 34 CASP-likes (CASPLs) in Arabidopsis, and CASPLs should have a conserved module for membrane subdomain formation and address different cell wall-modifying machineries in different tissues (Roppolo et al., 2014). There are 6 OsCASPs and 28 OsCASPLs in rice (Fig. S1), and the function of OsCASP1 has recently been studied, which indicates that OsCASP1 is required for CS formation in endodermal cells(Z. Wang et al., 2019). The rice root system is more complex than that of Arabidopsis, and its radial structure includes the epidermis, exodermis, sclerenchyma, midcortex, endodermis, and stele from the outside inward (Rebouillat et al., 2009). There is no CS of the exodermis, sclerenchyma, and aerenchyma inArabidopsis root (Rebouillat et al., 2009; Robbins II et al., 2014). These specified tissues allow rice to adapt to the growth condition.

Leaf senescence is caused by interplay between internal and external factors and is highly regulated by the coordinated actions of multiple pathways (Woo, Kim, Lim, \& Nam, 2019). An abnormal CS and ectopic suberin deposition cause an ion imbalance of aerial parts and leaf cell death (Table S1). Here we report the characterization of the Oscasp1 mutant, which shows obvious early leaf senescence (els1). OsCASP1 shows high similarity to AtCASPs (Fig. S1 and S2), but it regulates the deposition of suberin at small lateral roots (SLRs). In this report, we also provided sufficient evidences that indicated that OsCASP1 is not required for CS formation.

\section{Materials and Methods}

Constructions for the complementation test, tissue-specific expression, and subcellular localization of OsCASP1 protein

Plasmids were constructed using the In-Fusion cloning kit (Takara). To generate OsCASP1pro:OsCASP1 , $1128 \mathrm{bp}$ upstream of the OsCASP1 gene, including the whole intergenic sequence between Os04g0684200 and Os04g0684200 (OsCASP1 ) and the OsCASP1gene were amplified, and the PCR product was recombined into pCAMBIA-1300 digested with Hind III with the In-Fusion cloning kit. To generate OsCASP1pro:OsCASP1-GUS andOsCASP1pro:OsCASP1-GFP, the promoter (including whole intergenic 
sequence) and $O s C A S P 1$ gene were amplified and recombined into pCXGUS-P and pCXGFP-P digested with $\mathrm{Xcm} I$, respectively. The constructs of 35S::DsRed-OsCASP1, 35S::DsRed-OsCASP1-N, and 35S::DsRedOsCASP1-C were generated by recombining theOsCASP1 gene into pCX-DR. The details of these constructs are shown in figure S3, and the primers used in this study were shown in table S4. The markers for the nucleus and endoplasmic reticulum were used for transient expression for the subcellular localization of OsCASP1 (Z. Chen et al., 2019).

For the complementation experiment, OsCASP1pro:OsCASP1 was transformed into a few $\mathrm{F}_{3}$ progenies of the els1mutant and Nipponbare, which exhibited fewer tillers and leaf cell death and looked like Nipponbare with respect to morphological phenotype. The constructs of OsCASP1pro:OsCASP1GUS ,OsCASP1pro:OsCASP1-GFP, and 35S::DsRed-OsCASP1 were transformed into Zhonghua 11. The CRISPR/cas9 mutant of OsCASP1 were generated by the Biogle Company, and the target sequence was localized to exon 1 of the $O s C A S P 1$ gene.

\section{Growth Conditions}

Rice plants were grown under two different conditions: soil and hydroponics. For soil experiments, the $\mathrm{F}_{2}$ and $\mathrm{F}_{3}$ populations of the cross of the els1 mutant and Nipponbare were grown in the experimental paddy field in Putian, Fujian province. Rice plants were grown in a growth cabinet under $28^{\circ} \mathrm{C} / 14$-h light and $28^{\circ} \mathrm{C} / 10$-h dark photoperiod in nutrient solution (Table S2) to test the sensitivity of the plants to nutrient deficiency.

\section{DNA and Protein Sequence Analysis}

The new molecular markers were designed according to the Nipponbare sequence, and the CASP and CASPlike proteins in rice were obtained by searching the RAP-DB database with the BlastP programmes. The Molecular Evolutionary Genetic Analysis programme (MEGA X) was used to generate phylogenetic trees using the maximum likelihood method and JTT matrix-based model (Kumar, Stecher, Li, Knyaz, \& Tamura, 2018).

\section{Histochemical staining}

Roots of $10^{\sim} 14$-d-old seedling growing in nutrient solution were used for the study, and freehand crosssections were cut at different regions. To observe the Casparian strip, root cross-sections were stained with $0.1 \%(\mathrm{w} / \mathrm{v})$ berberine chloride and 0.5\% (w/v) aniline blue as described by Brundrett et al. (1988)(Brundrett, Enstone, \& Peterson, 1988). Casparian strips were visualized as bright white/yellow fluorescence (UV filter set). To visualize lignin with cinnamyl aldehyde groups in the roots, cross-sections were stained for 30 min with $1 \%$ phloroglucinol in $20 \%(\mathrm{w} / \mathrm{v})$ hydrochloric acid at room temperature. Lignin appears orange/red under white light (Pradhan Mitra \& Loque, 2014; Shiono et al., 2014). For the periodic acid staining, root cross-sections were incubated in $0.1 \%(\mathrm{w} / \mathrm{v}) \mathrm{H}_{5} \mathrm{IO}_{6}$ for 60 min and washed with reducing solution ( $1 \mathrm{~g}$ of KI and $1 \mathrm{~g}$ of $\mathrm{Na}_{2} \mathrm{~S}_{2} \mathrm{O}_{3} \cdot 5 \mathrm{H}_{2} \mathrm{O}$ dissolved in $50 \mathrm{ml}$ of $\mathrm{H}_{2} \mathrm{O}$ and acidified with $0.5 \mathrm{ml}$ of $2 \mathrm{M} \mathrm{HCl}$ ) for $60 \mathrm{~min}$. The cross-sections were visualized as purple staining in the cell walls under a light microscope (Shiono et al., 2014). Suberin deposition in the roots was visualized with Fluorol Yellow 088 as described by Barberon et al.(Barberon et al., 2016).

\section{Electron Microscopy}

The state I endodermis was visualized using root sections collected $5^{\sim} 10 \mathrm{~mm}$ from the root tips in 17 -d-old seedlings. Transmission electron microscopy (TEM) was conducted using a previously described protocol (Hulskamp, Schwab, Grini, \& Schwarz, 2010). 


\section{Permeability test}

For the propidium iodide penetration assay, the roots of $8 \sim 13$-d-old seedlings were incubated in the dark for $40 \mathrm{~min}$ in a fresh solution of $15 \mu \mathrm{M}(10 \mu \mathrm{g} / \mathrm{ml})$ propidium iodide and rinsed two times in water. They were then visualized with a confocal laser-scanning microscope(Naseer et al., 2012). The permeability of the exodermal and endodermal layers of roots was assessed with periodic acid and berberine as described by Shiono et al.(Shiono et al., 2014)

\section{Elemental Analysis}

The leaves in the tillering period were collected and used for elemental analysis, among which the els1 mutant showed a few cell death dots. The tissues were completely dried in an oven, and the samples were treated as described by Hosmani et al (2013)(Hosmani et al., 2013; Z. Wang et al., 2019). Elemental analysis was performed on a CIC-260 ion chromatograph (K, Na, Mg, and Ca) and ICP-MS (Cd, As, Mo, Fe, and Mn), and nine elements were monitored.

\section{RNA-seq}

The second and third leaves from the top at the tillering stage were collected separately. There was an obvious cell death phenotype in the third leaf and no visible cell death phenotype in the second leaf at this stage in the els1 mutant (Fig. S19A). Each treatment included two biological repeats. Total RNA was extracted using the method of Bilgin et al.(Bilgin, DeLucia, \& Clough, 2009) and then sent to Novogene company (service@novogene.com) for RNA-seq analysis. The resulting sequence data including 33.8 million 42.6 million paired-reads to each sample was provided for preliminary analysis. The differentially expressed genes (DEGs) were revealed with the DeSeq programme(Andersen, Barberon, \& Geldner, 2015), and the

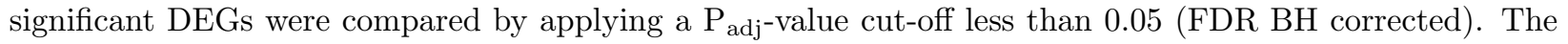
enrichment of DEGs in the gene ontology was analysed with g:Profiler (https://biit.cs.ut.ee/gprofiler/gost), and the enrichment in the KEGG pathway was revealed with KOBAS (2.0)(Xie et al., 2011). The $\mathrm{P}_{\text {adj-value }}$ denotes the significance of a GO term enrichment in DEG clusters and/or pathway correlations ( $\mathrm{P}_{\text {adj-value }}$ $<=0.05$ was considered significant).

\section{Results}

\section{Characterization of the rice els 1 mutant and cloning of the ELS1 gene}

The els1 mutant was discovered in a paddy field and came from a high generation progeny of Jinhui2629 and TR-2. The mutant and wild-type (WT) used in the study showed stable inheritance. The phenotype of the els1 mutant appears at the beginning of the tillering stage and is not obvious before this stage. Cell death usually begins at the blade tip or upper part of the leaf and then expands through the whole leaf along with growth. The els1 mutant exhibits fewer tillers and a reduced plant height at the adult stage (Fig. $1 \mathrm{~A}^{\sim} \mathrm{C}$ and Fig. S4A $\sim$ ).

To reveal the genetic basis of els1 mutant, we performed a cross between the els1 mutant and Nipponbare and investigated 426 individual plants from the $\mathrm{F}_{2}$ population. Genetic analysis showed that the els1 mutant was controlled by a single recessive gene. We mapped the els1 locus on chromosome 4 and delimited the locus to approximately a 265 -kb region between the insertion-deletion (Indel) molecular markers ID4-3 and ID4-10 using the $\mathrm{F}_{2}$ population. To further fine-map the els1 gene, we selected several $\mathrm{F}_{2}$ individual plants with a heterozygous genotype in the candidate region to establish the $\mathrm{F}_{3}$ population. The els1 locus was narrowed down to an 11.3-kb region between ID4-371 and ID4-3-8 using Indel markers, which included two genes - Os04g0684200 and Os04g0684300 (Fig. S4E). We sequenced the two genes and found a 15-bp deletion in the coding region of the Os04g0684300 gene in the els1 mutant and no mutation in the Os04g0684200 gene (Fig. 1D). Os04g0684300 encodes a Casparian strip membrane domain protein (CASP1), and the mutation of Os04g0684300 could induce early leaf senescence. 
To further validate CASP1, we performed genetic complementation by introducing the OsCASP1 gene with its promoter into the callus from $\mathrm{F}_{3}$ seeds, which arose from a few individuals from the $\mathrm{F}_{2}$ population that exhibited mutant and Nipponbare-like phenotypes to reduce transformation difficulty. The results showed that all transgenic lines carrying the OsCASP1gene had a restored WT phenotype (Fig. $1 \mathrm{E}$ and $1 \mathrm{~F}$ ). We also generated transgenic plants to knock out the OsCASP1 gene with CRISPR-cas9 technology, and all the transgenic plants with the homozygous genotype exhibited obvious early leaf senescence, sterility or eventual death (Fig. S5). We found that some transgenic plants with the heterozygous genotype also showed mutant phenotypes, and further analysis revealed that both alleles at the OsCASP1 locus contained mutant sites (Fig. S5).

\section{OsCASP1 was mainly localized on the nuclear membrane}

To determine the subcellular localization of OsCASP1, we generated anOsCASP1pro:OsCASP1-GFP construct and transformed it into Zhonghua 11. Unexpectedly, we observed that the green fluorescence was concentrated in the nucleus in the lateral rootlet tips (Fig. 2A). The localization of OsCASP1 differed from that of AtCASPs, which was present at the CSD in the endodermis. We also noticed some green fluorescence on the plasma membrane in the main and lateral root elongation zones, but it was difficult to distinguish the fluorescence from GFP or autofluorescence (Fig. S6A). Thus, we transformed 35S::DsRed-OsCASP1 and OsCASP1pro:OsCASP1-GFP into tobacco, onion and Arabidopsis protoplast, respectively. All of these results indicated that OsCASP1 protein was mainly localized to the nucleus (Fig. 2B and Fig. S6). Because the protein contains transmembrane domains, we speculated that OsCASP1 should be localized to the nuclear membrane. Moreover, the fluorescence was also detected on the plasma membrane in tobacco and onion, albeit with a much weaker signal (Fig. 2B, Fig. S6B $\sim$ D). This result implied that the protein could be located on the plasma membrane. To reveal whether OsCASP1 was present on the plasma membrane in rice, we transformed $35 S:: D s R e d-O s C A S P 1$ into Zhonghua 11 and detected red fluorescence on the plasma membrane in the small lateral root (Fig. S7A). We observed strong red fluorescence in the nuclei and some other intracellular compartments in roots (Fig. S7), which could be endoplasmic reticulum attached to the nucleus. Additionally, OsCASP1 protein aggregated in this region due to overexpression of this gene (Fig. $\mathrm{S} 6 \mathrm{E}$ and S7D). The strong red fluorescence was concentrated in the stele of roots (Fig. S7C and S7E) and did not restrict the localization within the CS domain.

Because the cellular position of OsCASP1 differed from that of AtCASPs, and OsCASP1could contain the fifth transmembrane according to the TMHMM prediction (Sonnhammer, von Heijne, \& Krogh, 1998) (Fig. $\mathrm{S} 2$ ), we generated two constructs containing the N-terminal region (1 50) and C-terminal region (62-224) of CASP1, respectively, and transformed them with the nuclear marker and the endoplasmic reticulum marker into rice protoplasts. We compared the protoplast cells of $35 S:: D s R e d-O s C A S P 1-N$ with that of $35 S:: D s R e d$ and discovered no obvious difference. In addition, the red fluorescence from DsRed-OsCASP1-N did not completely overlap with the green fluorescence from the endoplasmic reticulum marker (Fig. S6F). These results indicated that OsCASP1 did not possess the fifth transmembrane domain. Further experiment revealed that the C-terminal region with four transmembrane domains was also localized in the nuclear membrane and endoplasmic reticulum similar to whole OsCASP1 protein. This result suggested that the localization signal was in the C-terminal region of OsCASP1 (Fig. 2 and Fig. S6).

\section{Pattern and induction of $O s C A S P 1$ gene expression.}

To detect the expression pattern of OsCASP1, we transformed OsCASP1pro:OsCASP1-GUS into Zhonghua 11 and observed very strong OsCASP1 expression at the tips of small lateral roots (SLRs) and weak expression in other regions of the roots but not all tips of SLRs. Salt stress strongly induced OsCASP1 expression in roots and leaves. We examined the cross and longitudinal sections of primary root after staining and found that GUS activity was mainly concentrated in the stele of roots. We also discovered OsCASP1 gene expression in other root tissues treated with $\mathrm{NaCl}$, especially sclerenchyma cells (Fig. 3 and Fig. S8). We could not detect OsCASP1 expression in leaves at the adult stage using GUS staining method and RT-PCR. 


\section{No significant difference of the primary root structure between els1 and WT}

The upper results suggested that the function of OsCASP1 should differ from that of AtCASPs. To reveal the function of OsCASP1 in the root, we first checked the structures of the primary roots (embryonic crown roots, postembryonic crown roots) and large lateral roots of theels1 mutant by separate staining with phloroglucinol, berberine-aniline blue, and periodic acid. Though we observed the cross-sections from different zones of many primary roots and/or large lateral roots, we found no observe obvious differences in the root structure between the WT and els1 mutant, including the CS structure (Fig. S9 and S10). These experiments were repeated many times, and we found no significant difference in the endodermis and exodermis between the WT and els1 mutant. To reveal the fine structures of CSs, we used electron microscope to observe the sections of primary roots and found no difference between the CSs of els1 and WT. We also checked the CS after treatment with salt stress and still found no CS difference between the mutant and WT (Fig. 4A).

We evaluated the suberin deposition at different zones of primary roots stained with Fluorol Yellow 088 in 7-day-old, 28-day-old, and 35-day-old seedling, respectively. Because there were considerable differences among roots in WT or the els1 mutant, we could not obtain a consistent result that indicated a significant difference in suberin formation between our mutant and WT.

\section{The small lateral roots of the els1 mutant display ectopic suberin deposition}

We then evaluated suberin deposition in SLRs with Fluorol Yellow 088. The results showed that the patterns of suberin deposition depended on the position of SLRs in the primary root. The newborn lateral roots near the primary root tip $\left(2^{\sim} 3 \mathrm{~cm}\right.$ behind the root apex) usually had no suberin deposition, and the SLRs without suberin deposition occurred more frequently in the WT than in the mutant (Fig. 4C). The SLRs that were far from the primary root tip in WT usually exhibited even colouring or ectopic deposition in a few cells. This result indicated that suberin deposition was evenly distributed along the SLRs in the WT. However, most SLRs in els1 showed ectopic deposition and an uneven distribution along the SLRs, and suberization increased along with root growth. Suberin deposition in SLRs of the WT was sensitive to environmental conditions, and SLRs among different seedlings showed greater differences in suberin deposition (Fig. 4B, 4C, and Fig. S11). To determine the position of ectopic suberin deposition, we observed the cross-sections of the SLRs and found that ectopic suberin deposition mainly occurred in endodermal cells in theels1 mutant (Fig. 4D and Fig. S12). We also observed strong fluorescence in the sclerenchyma, which could allow the SLRs of WT to exhibit continuous fluorescence (Fig. 4D and Fig. S13). We were not sure whether the strong fluorescence of sclerenchyma arose from autofluorescence and/or suberin stained with Fluorol Yellow 088, but the sclerenchyma could be coloured with Sudan Red 7B, which is also used to stain suberin(Brundrett, Kendrick, \& Peterson, 1991; Schreiber, Franke, Hartmann, Ranathunge, \& Steudle, 2005). We observed the SLRs stained with berberine-aniline blue and discovered patchy white-blue zones from endodermal cells in the els1 mutant, as observed with Fluorol Yellow 088. However, the SLRs of the WT showed continuous white-blue fluorescence, which was strong or weak. This fluorescence could arise from both lignin and suberin stained with berberine-aniline blue and mainly from sclerenchyma cells of WT (Fig. S13).

\section{The apoplastic barrier function ofels1 roots}

Propidium iodide (PI) was used as an apoplastic tracer to reveal the functional apoplastic barrier in roots of Arabidopsis (Alassimone, Roppolo, Geldner, \& Vermeer, 2012), thus we applied this substance to rice. The results showed that the CS of WT rice could not hinder PI entry into the stele. However, different zones of the primary root showed different permeabilities to PI, and the zone far from the root tip exhibited more retardation of PI. This retardation did not seem to come from CS in exodermis and endodermis (Fig. S14A). The PI concentration $(10 \mu \mathrm{g} / \mathrm{ml})$ used to stain the roots of Arabidopsis did not seem to be sufficient for the primary roots of rice, which often causes only partial staining of transverse sections (Fig. S14A). We attempted to use a higher concentration of PI $(500 \mu \mathrm{g} / \mathrm{ml})$ and found that whole root was uniformly coloured 
(Fig. S14C). These results indicated that the primary roots could not completely block the penetration of PI into stele. We also detected the SLRs with the lower concentration PI, and the result suggested that the stele of root was strongly coloured. In addition, there was no difference in permeability to PI between the WT andels1 mutant (Fig. S14B). These results suggested that the CS structure and function of rice were different from those of Arabidopsis .

Periodic acid and berberine were used as apoplastic tracers in rice, and they were blocked at the outside of the exodermis of the WT roots under stagnant deoxygenated conditions (Shiono et al., 2014). We detected the permeability of CS with berberine chloride and periodic acid and found that the different root regions showed different permeabilities to berberine and periodic acid, and the region close to the root tip exhibited stronger permeability. However, we did not discover significant differences between els1 and WT (Fig. S10 and S15). We detected the root permeability by staining with phloroglucinol for different lengths of time and found that the staining of sclerenchyma and stele cells was stronger in the WT than the mutant (Fig. S16). The results indicated that the permeability of the exodermis and endodermis to phloroglucinol might be lower in the mutant than in the WT.

The els1 mutant displays ion homeostasis defects and different sensitivities to different nutrient stresses

Mutation of $O s C A S P 1$ changed the deposition of suberin in SLRs and probably altered the ion permeability of the mutant. We measured the content of 9 metal elements in leaves in the tillering period, in which cell death partly appeared. We found a significant accumulation of iron, manganese, and sodium and a reduction in potassium and arsenic in the els1 mutant (Fig. S17). The results indicated that the OsCASP1 mutation altered ion uptake in the root, and the report by Wang et al. (2019) also indicated that the shoots of theOscasp1 mutant display nutrient homeostasis defects (Z. Wang et al., 2019). We speculated that the disorder of ion homeostasis in plants resulted in the mutant phenotype, and then we tested the growth of els1 plants in nutrient-poor solution in a climate incubator and found that the mutant displayed distinct phenotypes in different stress conditions. There was no visible leaf cell death in the els1 mutant in complete medium. The mutant was insensitive to the deficiency of phosphorous, iron, or nitrogen and to a high concentration of phosphorous or iron (data not shown), and there were slight differences in the leaves betweenels1 and WT in medium without potassium, magnesium, or aluminium (Fig. $5 \mathrm{~A}^{\sim} \mathrm{C}$, and Fig.S18A $\sim$ C). The WT showed early senescence of lower leaves in medium without magnesium or with aluminium (Fig. 5B, 5C, Fig. S18B, and S18C). WT plants were sensitive to the medium with a low pH value $(\mathrm{pH}=4.0)$ and showed no obvious differences in tillers under this condition from the mutant (Fig. 5D and Fig. S18D). In addition, the mutant showed curled and dry leaves in the medium with cadmium (Fig. S18E) and was sensitive to high concentrations of $\mathrm{NaCl}$ (Fig. 5E). These results suggested that the els1 mutant exhibited different sensitivity to different nutrient stresses, which could be relevant to the composition of mineral ions in the mutant plant.

\section{The function of the chloroplast is depressed and nutrient recycling is enhanced in the els1 mutant}

To reveal the mechanism that regulates cell death, we performed RNA-seq with the second and third leaves from the top, respectively. There was no obvious cell death phenotype in the second leaf and an obvious phenotype in the third leaf in the els1 mutant (Fig. S19A). We separately found 2752 and 1668 differentially expressed genes (DEGs) in second and third leaves between the WT and els1 mutant (Fig. S19B). To achieve a functional annotation of the genes related to leaf cell death, we performed GO enrichment analysis. The significantly enriched DEGs were mainly concentrated in the chloroplast and downregulated in the els1 mutant compared with the WT. Accordingly, the DEGs related to photosynthesis as a biological process and electron transfer activity as a molecular function were significantly enriched (Table S3). In addition, downregulation of genes involved in the response to light stimulus were enriched in the mutant, and most proteins encoded by these genes were located in the chloroplast. KEGG pathway enrichment analysis revealed that the DEGs encoding proteins involved in the light-harvesting chlorophyll protein complex, electron transport chain and ATP-synthesizing apparatus in the thylakoid membrane and carbon fixation of photosynthesis 
were downregulated (Fig. S19). We also noticed that the DEGs involved in ion homeostasis and transport biology process and ion binding molecular function were also significantly enriched and upregulated in the second leaf of the mutant (Table S3). All these results implied that the ion imbalance in the mutant could induce autophagy and nutrient recycling and mobilization from the lower leaf to sink tissues. We then checked the DEGs and found that some autophagy-related genes (ATGs) were upregulated in the mutant and significantly enriched in macroautophagy, which encode key components for autophagosome formation and participate in autophagic process(Q. Chen et al., 2019; Have, Marmagne, Chardon, \& Masclaux-Daubresse, 2017) (Fig. S20A). The changes in expression of these genes and repression of chloroplast function indicated that autophagy was initiated in the second leaf of the mutant. Nutrient recycling also requires various proteases and transporters, and chloroplast material and unwanted cytoplasmic material are driven to the vacuole by autophagosome for degradation by proteases and hydrolases, and the released nutrients (including amino acids, organic acids, glyceride, and mineral elements) are exported to the cytosol and then transported to newer tissue via transporters(Q. Chen et al., 2019). Thus, we checked the DEGs of proteases, proteases inhibiters, transporters, and channels. The genes of proteases and proteases inhibiter were selected according to figure 2 and table S1 published by Have et al. (2019), which are orthologues of these Arabidopsisgenes(Q. Chen et al., 2019). The results showed a significant enrichment of the genes encoding aspartic-type peptidase, cysteine-type peptidase, serine-type peptidase, and metallopeptidase and the peptidases in the vacuole and chloroplast envelope (Fig. S20B ${ }^{\sim} \mathrm{D}$ ), which take part in chloroplast dismantling (Q. Chen et al., 2019; Have et al., 2017). There was an enrichment of genes encoding anion transporters (including amino acids, phosphate, and sulphate transporters), cation transporters (including metal ion transporter), channels, and solute:cation symporters, which participate in nutrient remobilization (Q. Chen et al., 2019; Have et al., 2017) (Fig. S21). These results suggested that nutrient starvation in the mutant plants resulted in nutrient recycling and accelerated lower leaf senescence.

\section{The mechanism of early leaf senescence in the els1 mutant}

We carefully checked the DEGs and their orthologs in Arabidopsisto reveal genes that directly regulate leaf senescence and in which mutations result in leaf cell death. Many of these DEGs were uncovered, even in the second leaf, which implied that cell death was initiated in the second leaf. Further analysis showed that these DEGs could be divided into two groups, and the changes in gene expression in group 1 could cause leaf cell death in the mutant. The effects of the genes in group 2 were opposite to those in group 1, which showed expression in the mutant that should prevent cell death (Fig. 6)(Bruggeman, Raynaud, Benhamed, \& Delarue, 2015; Leng, Ye, \& Zeng, 2017; Woo et al., 2019). It was difficult to understand the changes in gene expression in group 2, which could be induced or repressed by leaf cell death. These and the above results implied that the metabolism in the mutant maintained a fragile balance under the right conditions, and the balance was easily broken by some adverse environmental factors, such as nutritional stress, which should result in dominance of the genes in group 1 leading to early leaf senescence.

More than half of the genes in group 1 encoded transcription factors (TFs), and most of the transcription factors belonged to NAC TFs. Among them, overexpression of OsNAP genes caused early leaf senescence, and suppression of the gene expression delayed leaf senescence (Liang et al., 2014; Zhou et al., 2013). AtNAP binds to the promoter of AtSAG113 (protein phosphatase 2C) and regulates gene expression, and SAG113 in turn inhibits stomatal closure in leaves, which results in faster water loss and consequently triggers leaf senescence(Zhang \& Gan, 2012).OsNAP and OsSAG113 genes were significantly upregulated in the els1 mutant (Fig. 6A and 8C), and OsNAP should use the same route to modulate leaf senescence and regulate chlorophyll breakdown. Chlorophyll breakdown is widely used as a tool for monitoring physiological senescence in plants. The expression of genes involved in chlorophyll breakdown, such as ACD1 (OsPAO), ACD2(OsRCCR1), SGR1 (OsSGR), AND PPH(OsNYC3), was directly regulated by OsNAP/AtNAP, AtORE1, and AtNAC019/055/072(Liang et al., 2014; Woo et al., 2019). All of these genes were upregulated in the els1 mutant, which accelerated the degradation of chlorophyll a (Fig. 6) and could result in chlorosis and cell death in the mutant leaves. In addition, these overexpressed NAC-TFs result in cell death, and the expression levels of these genes influence a variety of processes associated with the induction of senescence 
and modulate the expression of SAGs except upper genes(Woo et al., 2019).

\section{Discussion}

Recently, OsCASP1 has been characterized and was thought to be required for CS formation at endodermal cells (Z. Wang et al., 2019). To reveal the function of OsCASP1, three aspects of research were needed. The first concerns the CS structure of theOscasp1 mutant, which was thought to be different from that of the WT based on the photographs of confocal laser-scanning microscope (Z. Wang et al., 2019). However, our transmission electron micrographs displayed more clear structure of CS and showed no difference between the mutant and WT (Fig. 5A). The second concerns the localization of OsCASP1, which was considered to accumulate at the CSD of rice roots and on the cell membrane in protoplast. This conclusion could be from the result of autofluorescence of CS and the oversight to the strong green fluorescing area at plasma membrane, which could be the nucleus (Z. Wang et al., 2019). Our results indicated that OsCASP1 was mainly located on the nuclear membrane. We also detected the protein on the cell membrane, especially in overexpressed transgenic plants, but the signal was weak. Moreover, its expression was concentrated at the SLR tip and in the root stele (Fig. 2, Fig. S6 and S7). The third concerns PI penetration, which was used to detect the permeability of the CS in Arabidopsis(Barberon, 2017; Hosmani et al., 2013; Naseer et al., 2012). However, our results indicated that PI was not suitable for detecting the CS permeability of rice. In short, OsCASP1 could not accumulate at the CSD and regulate CS formation.

Until now, there have been no reports showing a CASP or CASPL protein mainly localized on the nuclear membrane, and only one CASPL has been detected in the endoplasmic reticulum(Roppolo et al., 2014). In addition, $C A S P L$ genes can be expressed in tissues other than the endodermis(Roppolo et al., 2014), and $A t C A S P L 4 C 1$ is widely expressed in a variety of organs and is cold-inducible; its mutant shows elevated tolerance to cold stress(Yang et al., 2015).AtCASPL1D2 is exclusively expressed in suberized endodermal cell and could regulate the deposition of suberin induced by $\mathrm{NaCl}$ stress (Champeyroux et al., 2019). The function of OsCASP1 differs from that of AtCASPs and can appear similar to some AtCASPLs. OsCASP1 is strongly expressed in sclerenchyma cell and could regulate the suberin deposition of sclerenchyma (Figure S13), andOscasp1 mutant is more sensitive to upland condition(Z. Wang et al., 2019) and shows different tolerances to different nutrient stresses (Fig. 5 and S18). These result implied that OsCASP1 could play a unique role in the adaption to the growth condition. How OsCASP1 on the nuclear membrane functions is a new and interesting topic. We found that OsCASP1 could interact with some membrane transcription factors (data not shown), which suggested that OsCASP1 could regulate suberin deposition by modulating the activity of the transcription factors.

We found no obvious difference between Oscasp1 and WT in primary roots, but the recent report indicated that there was a clear distinction of suberin deposition between Oscasp1 and the WT by analyses of crosssections. Unfortunately, the conclusion lacked statistical support (Z. Wang et al., 2019). We observed many cross-sections from different zones of many primary and large lateral roots and could not obtain consistent results to support the difference. In Arabidopsis, the number of endodermal cells from the first fully expanded cell can be counted to quantitatively describe the formation of the CS, the deposition of suberin, and permeability to PI (Hosmani et al., 2013). We attempted to detect autofluorescence of CS with whole SLRs like Arabidopsis, but the fluorescence emitted by CS was masked by the lignified sclerenchyma cells, which were outside the endodermis in rice and emitted strong fluorescence. Until now, there has been no appropriate method to observe CS in SLRs using the whole mount method. However, we detected ectopic deposition of suberin in the mutant SLRs after staining with Fluorol Yellow 088 or berberine with whole SLRs. SLRs contain more root hairs than primary and large lateral roots and should be the main tissue for nutrient uptake. OsCASP1 mutation results in a nutrient imbalance in the plant (Fig. S17)(Z. Wang et al., 2019), which should be due to the ectopic suberin deposition in the SLRs.

Leaf senescence is a developmental process, in which cellular structures and biomolecules are progressively broken down to reallocate nutrients to juvenile and reproductive organs. This process enhances the chances of plant survival to adapt to biotic/abiotic stresses (Q. Chen et al., 2019). Nitrogen is the element that is 
predominantly remobilized during leaf senescence, along with potassium. Many other elements are also remobilized, although less efficiently than nitrogen (Have et al., 2017; Himelblau \& Amasino, 2001). OsCASP1 mutation leads to mineral element imbalance in the mutant shoot(Z. Wang et al., 2019), which enhances autophagy activity and accelerates nutrient recycling(Q. Chen et al., 2019). Our results indicated that the DEGs involved in nutrient recycling were significantly enriched, and the main functions of the chloroplast were depressed in the mutant, even in the second leaf, which showed no visible cell death phenotype. As state above, we rendered a model to address the mechanism of early leaf senescence in the Oscasp1 mutant (Fig. 7).

\section{ACKNOWLEDGMENTS}

We thank Songbiao Chen of Institute of Oceanography, Minjiang University, Fuzhou, China for providing vectors and protein-tagged organelle markers, and Fangyu Chen of College of Agriculture, Fujian Agriculture and Forestry University for helping in electron microscopy, and Zhu Li of NERCS of Fujian Agriculture and Forestry University for helping in transient expression.

This work was supported by grant from the National Natural Science Foundation of China (31571574)

\section{Author contributions}

X.S. conceived and designed the experiments, wrote the manuscript, and took part in some experiments; H.X. completed the map-based cloning ofels1 gene; X.H. and Q.W. did complementation test and CRISPR-cas9 of OsCASP1; X.Y. performed electron microscopy and suberin deposition analysis; Q.W., X.Y. and X.Z. did subcellular localization of OsCASP1; X.Y. and Q.W. performed histological analysis and hydroponic experiment. Q.W., X.Y., X.S., X.Z and H.X. carried out the analysis of OsCASP1 expression and RNA-seq. K.L and Y.G. performed field experiments and management. All authors commented on the manuscript.

Competing interests The authors declare no competing financial interests.

\section{Reference}

Alassimone, J., Roppolo, D., Geldner, N., \& Vermeer, J. E. (2012). The endodermis-development and differentiation of the plant's inner skin. Protoplasma, 249 (3), 433-443. doi:10.1007/s00709-011-0302-5

Andersen, T. G., Barberon, M., \& Geldner, N. (2015). Suberization-the second life of an endodermal cell.Curr Opin Plant Biol, 28 , 9-15. doi:10.1016/j.pbi.2015.08.004

Barberon, M. (2017). The endodermis as a checkpoint for nutrients. New Phytol, 213 (4), 1604-1610. doi:10.1111/nph.14140

Barberon, M., \& Geldner, N. (2014). Radial transport of nutrients: the plant root as a polarized epithelium.Plant Physiol, 166 (2), 528-537. doi:10.1104/pp.114.246124

Barberon, M., Vermeer, J. E., De Bellis, D., Wang, P., Naseer, S., Andersen, T. G., . . . Geldner, N. (2016). Adaptation of Root Function by Nutrient-Induced Plasticity of Endodermal Differentiation. Cell, 164 (3), 447-459. doi:10.1016/j.cell.2015.12.021

Bilgin, D. D., DeLucia, E. H., \& Clough, S. J. (2009). A robust plant RNA isolation method suitable for Affymetrix GeneChip analysis and quantitative real-time RT-PCR.Nat Protoc, 4 (3), 333-340. doi:10.1038/nprot.2008.249

Bruggeman, Q., Raynaud, C., Benhamed, M., \& Delarue, M. (2015). To die or not to die? Lessons from lesion mimic mutants. Front Plant Sci, 6 , 24. doi:10.3389/fpls.2015.00024

Brundrett, M. C., Enstone, D. E., \& Peterson, C. (1988). A berberine-aniline blue fluorescent staining procedure for suberin,lignin and callose in plant tissue.Protoplasma, 146 , 133-142. 
Brundrett, M. C., Kendrick, B., \& Peterson, C. A. (1991). Efficient lipid staining in plant material with sudan red 7B or fluorol [correction of fluoral] yellow 088 in polyethylene glycol-glycerol. Biotech Histochem, 66 (3), 111-116.

Champeyroux, C., Bellati, J., Barberon, M., Rofidal, V., Maurel, C., \& Santoni, V. (2019). Regulation of a plant aquaporin by a Casparian strip membrane domain protein-like.Plant Cell Environ, 42 (6), 1788-1801. doi:10.1111/pce.13537

Chen, Q., Shinozaki, D., Luo, J., Pottier, M., Have, M., Marmagne, A., . . . Masclaux-Daubresse, C. (2019). Autophagy and Nutrients Management in Plants. Cells, 8 (11). doi:10.3390/cells8111426

Chen, Z., Zheng, W., Chen, L., Li, C., Liang, T., Chen, Z., . . . Chen, S. (2019). Green Fluorescent Protein- and Discosoma sp. Red Fluorescent Protein-Tagged Organelle Marker Lines for Protein Subcellular Localization in Rice. Front Plant Sci, 10 , 1421. doi:10.3389/fpls.2019.01421

Doblas, V. G., Geldner, N., \& Barberon, M. (2017). The endodermis, a tightly controlled barrier for nutrients. Curr Opin Plant Biol, 39 , 136-143. doi:10.1016/j.pbi.2017.06.010

Have, M., Marmagne, A., Chardon, F., \& Masclaux-Daubresse, C. (2017). Nitrogen remobilization during leaf senescence: lessons from Arabidopsis to crops. J Exp Bot, 68 (10), 2513-2529. doi:10.1093/jxb/erw365

Himelblau, E., \& Amasino, R. M. (2001). Nutrients mobilized from leaves of Arabidopsis thaliana during leaf senescence. J Plant Physiol, 158 (10), 1317-1323. doi:10.1078/0176-1617-00608

Hosmani, P. S., Kamiya, T., Danku, J., Naseer, S., Geldner, N., Guerinot, M. L., \& Salt, D. E. (2013). Dirigent domain-containing protein is part of the machinery required for formation of the lignin-based Casparian strip in the root. Proc Natl Acad Sci U S A, 110 (35), 14498-14503. doi:10.1073/pnas.1308412110

Hulskamp, M., Schwab, B., Grini, P., \& Schwarz, H. (2010). Transmission electron microscopy (TEM) of plant tissues. Cold Spring Harb Protoc, 2010 (7), pdb prot4958. doi:10.1101/pdb.prot4958

Kumar, S., Stecher, G., Li, M., Knyaz, C., \& Tamura, K. (2018). MEGA X: Molecular Evolutionary Genetics Analysis across Computing Platforms. Mol Biol Evol, 35 (6), 1547-1549. doi:10.1093/molbev/msy096

Lee, Y., Rubio, M. C., Alassimone, J., \& Geldner, N. (2013). A mechanism for localized lignin deposition in the endodermis. Cell, 153 (2), 402-412. doi:10.1016/j.cell.2013.02.045

Leng, Y., Ye, G., \& Zeng, D. (2017). Genetic Dissection of Leaf Senescence in Rice. Int J Mol Sci, 18 (12). doi:10.3390/ijms18122686

Liang, C., Wang, Y., Zhu, Y., Tang, J., Hu, B., Liu, L., . . . Chu, C. (2014). OsNAP connects abscisic acid and leaf senescence by fine-tuning abscisic acid biosynthesis and directly targeting senescence-associated genes in rice. Proc Natl Acad Sci U S A, 111 (27), 10013-10018. doi:10.1073/pnas.1321568111

Nakayama, T., Shinohara, H., Tanaka, M., Baba, K., Ogawa-Ohnishi, M., \& Matsubayashi, Y. (2017). A peptide hormone required for Casparian strip diffusion barrier formation in Arabidopsis roots. Science, 355 (6322), 284-286. doi:10.1126/science.aai9057

Naseer, S., Lee, Y., Lapierre, C., Franke, R., Nawrath, C., \& Geldner, N. (2012). Casparian strip diffusion barrier in Arabidopsis is made of a lignin polymer without suberin. Proc Natl Acad Sci U S A, 109 (25), 10101-10106. doi:10.1073/pnas.1205726109

Pradhan Mitra, P., \& Loque, D. (2014). Histochemical staining of Arabidopsis thaliana secondary cell wall elements. J Vis Exp (87). doi:10.3791/51381

Rebouillat, J., Dievart, A., Verdeil, J. L., Escoute, J., Giese, G., Breitler, J. C., . . . Perin, C. (2009). Molecular Genetics of Rice Root Development. Rice (N Y), 2 (1), 15-34. doi:10.1007/s12284-008-9016-5

Robbins II, N. E., Trontin, C., Duan, L., \& Dinneny, J. R. (2014). Beyond the Barrier: Communication in the Root through the Endodermis. Plant Physiol, 166 (2), 551-559. doi:10.1104/pp.114.244871 
Roppolo, D., Boeckmann, B., Pfister, A., Boutet, E., Rubio, M. C., Denervaud-Tendon, V., . . . Geldner, N. (2014). Functional and Evolutionary Analysis of the CASPARIAN STRIP MEMBRANE DOMAIN PROTEIN Family. Plant Physiol, 165 (4), 1709-1722. doi:10.1104/pp.114.239137

Roppolo, D., De Rybel, B., Denervaud Tendon, V., Pfister, A., Alassimone, J., Vermeer, J. E., . . . Geldner, N. (2011). A novel protein family mediates Casparian strip formation in the endodermis. Nature, 473 (7347), 380-383. doi:10.1038/nature10070

Schreiber, L., Franke, R., Hartmann, K. D., Ranathunge, K., \& Steudle, E. (2005). The chemical composition of suberin in apoplastic barriers affects radial hydraulic conductivity differently in the roots of rice (Oryza sativa L. cv. IR64) and corn (Zea mays L. cv. Helix). J Exp Bot, 56 (415), 1427-1436. doi:10.1093/jxb/eri144

Shiono, K., Ando, M., Nishiuchi, S., Takahashi, H., Watanabe, K., Nakamura, M., . . . Kato, K. (2014). RCN1/OsABCG5, an ATP-binding cassette (ABC) transporter, is required for hypodermal suberization of roots in rice (Oryza sativa). Plant $J, 80$ (1), 40-51. doi:10.1111/tpj.12614

Sonnhammer, E. L., von Heijne, G., \& Krogh, A. (1998). A hidden Markov model for predicting transmembrane helices in protein sequences. Proc Int Conf Intell Syst Mol Biol, 6 , 175-182.

Wang, P., Calvo-Polanco, M., Reyt, G., Barberon, M., Champeyroux, C., Santoni, V., . . . Salt, D. E. (2019). Surveillance of cell wall diffusion barrier integrity modulates water and solute transport in plants. Sci Rep, 9 (1), 4227. doi:10.1038/s41598-019-40588-5

Wang, Z., Yamaji, N., Huang, S., Zhang, X., Shi, M., Fu, S., . . . Xia, J. (2019). OsCASP1 is Required for Casparian Strip Formation at Endodermal Cells of Rice Roots for Selective Uptake of Mineral Elements. Plant Cell .

Woo, H. R., Kim, H. J., Lim, P. O., \& Nam, H. G. (2019). Leaf Senescence: Systems and Dynamics Aspects.Annu Rev Plant Biol, 70 , 347-376. doi:10.1146/annurev-arplant-050718-095859

Xie, C., Mao, X., Huang, J., Ding, Y., Wu, J., Dong, S., . . . Wei, L. (2011). KOBAS 2.0: a web server for annotation and identification of enriched pathways and diseases. Nucleic Acids Res, 39 (Web Server issue), W316-322. doi:10.1093/nar/gkr483

Yang, J., Ding, C., Xu, B., Chen, C., Narsai, R., Whelan, J., . . . Zhang, M. (2015). A Casparian strip domain-like gene, CASPL, negatively alters growth and cold tolerance.Sci Rep, 5 , 14299. doi:10.1038/srep14299

Zhang, K., \& Gan, S. S. (2012). An abscisic acid-AtNAP transcription factor-SAG113 protein phosphatase 2C regulatory chain for controlling dehydration in senescing Arabidopsis leaves. Plant Physiol, 158 (2), 961-969. doi:10.1104/pp.111.190876

Zhou, Y., Huang, W., Liu, L., Chen, T., Zhou, F., \& Lin, Y. (2013). Identification and functional characterization of a rice NAC gene involved in the regulation of leaf senescence. BMC Plant Biol, 13 , 132. doi:10.1186/1471-2229-13-132

Figure 1. The phenotypes of the wild type andels1 mutant and characterization of els1.(A) Seedlings of wild type and els1 mutant plants. (B) The phenotypes of wild type and els1 mutant plants at the heading stage. Red arrows indicate leaves with necrosis. (C) Phenotype of the leaves. 1, 2, and 3 indicate the relative positions from top to bottom. (D) The structure of OsCASP1 and deletion region in the els1 mutant. (E) Phenotypic comparison of transgenic plants with the OsCASP1 gene (T6 and T4) and segregant individuals without theOsCASP1 gene (T1 and T2). (F) The PCR result indicated transgenic plants with or without the OsCASP1 gene.

Figure 2. Subcellular localization of OsCASP1 protein.

(A) OsCASP1pro:OsCASP1-GFP in rice root. (B) 35S::GFP and35S::OsCASP1-GFP in tobacco leaves.

(C) 35S::DsRed-CASP1-Cin rice protoplasts. 
Figure 3. Localization of OsCASP1pro:OsCASP1-GUSexpression.

(A) GUS activity in the rootlet tip. (B) and (C) GUS staining of whole seedling plants and plants treated with $100 \mathrm{mM} \mathrm{NaCl}$ for 3 hours. (D) Cross-section of a crown root (13 mm distal from the root tip) derived from a 20-d-old seedling treated with $\mathrm{NaCl}$. Scale bars: (A) $100 \mu \mathrm{m},(\mathrm{B})$ and (C) $1 \mathrm{~cm}$; (D) $100 \mu \mathrm{m}$.

Figure 4. The Casparian strip structures and patterns of suberin deposition of the roots.

(A) The representative transmission electron micrographs of CS in the WT, els1 mutant, the WT and els1 mutant treated with salt stress. The transverse sections arise from the zone of $5-10 \mathrm{~mm}$ far from root tip. 1, epidermis; 2, exodermis; 3, sclerenchyma; 4, endodermis. (B) The patterns of suberin deposition in small lateral roots of the WT and els1 mutant, which were obtained with laser confocal microscopy. Scale bar: $100 \mu \mathrm{m}(\mathrm{C})$ The percentage of small lateral roots of each deposition pattern. (D) Representative transverse sections from small lateral roots stained with FY088 were observed with laser confocal microscopy.

Figure 5. Phenotypes of WT and els1 mutant plants grown in various media with elemental mineral imbalances.

(A) In medium without potassium. (B) In medium without magnesium. (C) Treatment with $100 \mu \mathrm{M} \mathrm{AlCl}_{3}$. (D) In medium with a low $\mathrm{pH}$ value $(\mathrm{pH}=4.0)$. (E) Phenotypes of WT and els1 mutant plants treated with $100 \mathrm{mM} \mathrm{NaCl}$. (F) In medium without iron under continuous light. (G) In medium without calcium under continuous light. (H) In medium without microelements under continuous light. Arrows indicate the different phenotypes between WT and els1 mutant plants.

Figure 6. The expression of genes that regulate leaf senescence.

(A) and (B) Heatmap of DEGs in group 1 (A) and group 2 (B), and the gene ID of rice, gene name, and names of their orthologues inArabidopsis, and their possible functions are indicated. (C) The qPCR results for some representative genes, ${ }^{* *}$ indicates a significant difference in the second or third leaf between els1 and WT. (D) The chlorophyll a degradation pathway, and enzymes and transcription factors in the pathway.

Figure 7. A schematic working model for regulation mechanism of OsCASP1.

Supplementary materials

Figure S1. The maximum-likelihood tree of OsCASP1 homologs with their protein sequences. The scale bar indicates the simple matching distance. The alignment and phylogenetic tree were constructed with MEGAX (http://megasoftware.net/). The numbers for interior branches indicate the bootstrap values for 500 replications.

Figure S2 . (A) Alignment of CASPs in Arabidopsis and rice. IC: Intracellular loop; TM: Transmembrane domain; EC: extracellular loop. (B) (C) Transmembrane domains of OsCASP1 was predicted with TMHMM 2.0 (http://www.cbs.dtu.dk/services/TMHMM/) (B) and Protter (http://wlab.ethz.ch/protter/\#) (C)

Figure S3. Models of the constructs for complementation test, tissue-specific expression, and subcellular localization of OsCASP1.CASP1-N includes a transmembrane region predicted by TMHMM with a lower probability. TM: Transmembrane region.

Figure S4. The phenotypes of the WT and els1 mutant and characterization of els1.(A) Internode length and plant height; IN = internode. (B) The tiller number of the WT and els1 mutant at the heading stage. (C) and (D) The tiller phenotype of the seedling at 33-d-old. The emergence of tiller in the els1 mutant was delayed. (E) The els1 locus was fine-mapped on chromosome 4. The numbers of recombinants are shown in brackets.

Figure S5. The genotype and phenotype of CRISPR/cas9 mutants.(A) Schematic map of OsCASP1 gene and sequence alignment of the sgRNA target region showing altered bases in different mutant lines. The target region of the sgRNA was indicate with arrow, and the heterozygous transgenic plants with different mutant sites were marked with rectangles. (B) The typical phenotypes of CRISPR/cas9 transgenic plants. 
Figure S6. Subcellular localizations of OsCASP1 protein. (A)OsCASP1pro:OsCASP1-GFP in rice root. (B) and (C)OsCASP1pro:OsCASP1-GFP in onion and Arabidopsisprotoplast. (D) and (E) 35S::DsRed-OsCASP1 in onion andArabidopsis protoplast. Circle indicates the nuclear position. (F) 35S::DsRed-CASP1-N in rice protoplasts. CASP1-N includes a transmembrane region predicted by TMHMM with a lower probability. ER: Endoplasmic reticulum.

Figure S7. Subcellular localizations of OsCASP1 protein in transgenic plant roots carrying 35S::DsRed-OsCASP1. (A) OsCASP1 in the cell membrane in small lateral roots. (B) and (C) OsCASP1 is concentrated in the stele of lateral roots. (D) and (E) OsCASP1 in main roots. Arrows indicate the nuclei.

Figure S8. The localization of OsCASP1pro:OsCASP1-GUSexpression after salt treatment. (A) Whole roots treated with NaCl. (B) Crown root with lateral roots. (C) Lateral root. (D) Transection of the crown root. (E) Longitudinal section of the crown root. ep, epidermis; ex, exodermis; sc, sclerenchyma; co, cortex; en, endodermis; pe, pericycle. Scale bar: (A) $1 \mathrm{~cm}$; (B), (C), (D) and (E) $0.1 \mathrm{~cm}$.

Figure S9. The representative cross-sections at different zones in primary roots stained with berberine-aniline blue and phloroglucinol. (A) Staining with berberine-aniline blue. Autofluorescence of cell walls is detected as blue. Arrow head indicates CS at endodermis. (B) Staining with phloroglucinol. Arrow head indicates endodermis. ep, epidermis; ex, exodermis; sc, sclerenchyma.

Figure S10. The representative cross-sections stained with periodic acid (A) and permeability of CS was evaluated using periodic acid (B). Purple color indicates that the periodic acid penetrated into root tissues. (C) The roots stained with periodic acid.

Figure S11. The representative patterns of the suberin deposition of the SLRs.

Figure S12. The enlargement of the suberin deposition in the SLRs. 1, epidermis; 2, exodermis; 3 , sclerenchyma; 4, endodermis.

Figure S13. The representative cross-sections at differentiation zones of small lateral roots and images of the whole SLRs stained with berberine-aniline blue. Autofluorescence of cell walls is detected as blue. 1, epidermis; 2, exodermis; 3, sclerenchyma; 4, endodermis. Red line indicate the position of cross-section.

Figure S14. The permeabilities of the exodermis and endodermis were evaluated using PI. Red fluorescence indicates that PI penetrated into root tissues. (A) The transverse sections from different zones of primary roots stained with lower concentration PI. The section positions from the root tip were marked at the left. Scale bar: $75 \mu \mathrm{m}$ (B) The transverse sections of small lateral roots stained with lower concentration PI. (C) The transverse sections from different zones of primary roots stained with higher concentration PI.

Figure S15. The permeabilities of the endodermis were evaluated using berberine-aniline blue. Autofluorescence of cell walls is detected as blue. Arrow head, endodermis.

Figure S16. The permeabilities of the exodermis and endodermis were evaluated using Phloroglucinol. Purple color indicates that the periodic acid penetrates into root tissues. ep, epidermis; ex, exodermis; sc, sclerenchyma; pe, pericycle; en, endodermis.

Figure S17. Mineral elemental content in the leaves of the WT and els1 mutant ( $\mathrm{g} / \mathrm{kg}$ Dry weight). ${ }^{*}$ and $* *$ indicate the significant differences between the WT and els1 mutant at $5 \%$ and $1 \%$ level, respectively.

Figure S18. Phenotypes of WT and els1 mutant plants grown in various media with nutrient imbalances. (A) In complete medium (CK) and without potassium (-K). (B) In medium without magnesium $(-\mathrm{Mg})$. (C) Treatment with $100 \mathrm{uM} \mathrm{AlCl}_{3}$. (D) In medium with low $\mathrm{pH}$ value $(\mathrm{pH}=4.0)$. (E) in medium with cadmium (80uM).

Figure S19. Heatmaps of z-score-normalized expression of genes with FDR $<0.05$ between the WT and els1 mutant leaves and pathways with the differentially expressed genes according 
to KEGG.(A) The second and third leaves from the top (marked with arrows) were collected for RNA-seq analysis, respectively. There is no obvious cell death phenotype in the second leaf and an obvious phenotype in the third leaf in the els1 mutant. 2: The second leaf from the top. 3: The third leaf from the top. WT: Wild type. (B) Venn diagram. M2 and M3: The second and third leaf of the mutant; WT2 and WT3: The second and third leaf of the WT. (C) Heatmap of the DEGs encoding antenna protein. (D) The lightharvesting chlorophyll protein complex with antenna proteins marked in green color. (E) Heatmap of the DEGs encoding proteins in the electron transport chain and the ATP-synthesizing apparatus in the thylakoid membrane. (F) The electron transport chain and ATP-synthesizing apparatus in the thylakoid membrane in chloroplast. $(\mathrm{J})$ Heatmap of the DEGs in carbon fixation of photosynthesis. (K) The genes encoding enzymes in C3 carbon fixation pathway. The green gene ID markers indicate that the gene expressions are higher in the WT than in the mutant.

Figure S20. Heatmaps and GO analyses with DEGs $(\mid \log 2($ Fold Change els2/WT) $\mid>1$ or Padj [?] 0.05) between the WT and els1. (A) Heatmap with DEGs of ATG genes. (B) Heatmap with DEGs of protease genes. (C) GO enrichment analysis of biological processes, cellular component, and KEGG with protease genes. (D) GO enrichment analysis of molecular activity with protease genes.

Figure S21. Heatmap and GO enrichment analyses with DEGs $(\mid \log 2$ (Fold change els1/WT)| $>1$ or Padj [?] 0.05) between els1 and WT. (A) Heatmap of DEGs of transporter and channel genes. (B) GO enrichment analysis of cellular component with transporter and channel genes. (C) Molecular activity. (D) Biological processes.

Table S1. The genes that regulate Casparian strip (CS) formation and suberin deposition in Arabidopsis .

Table S2. Formula of hydroponic nutrient solution used in this study $(\mathrm{pH}=5.8$ ).

Table S3. Comparative gene ontology (GO) enrichment analysis of differentially expressed genes (DEGs) between Oscasp1 mutant and wild type-cellular component.

Table S4: The primers used in this study.
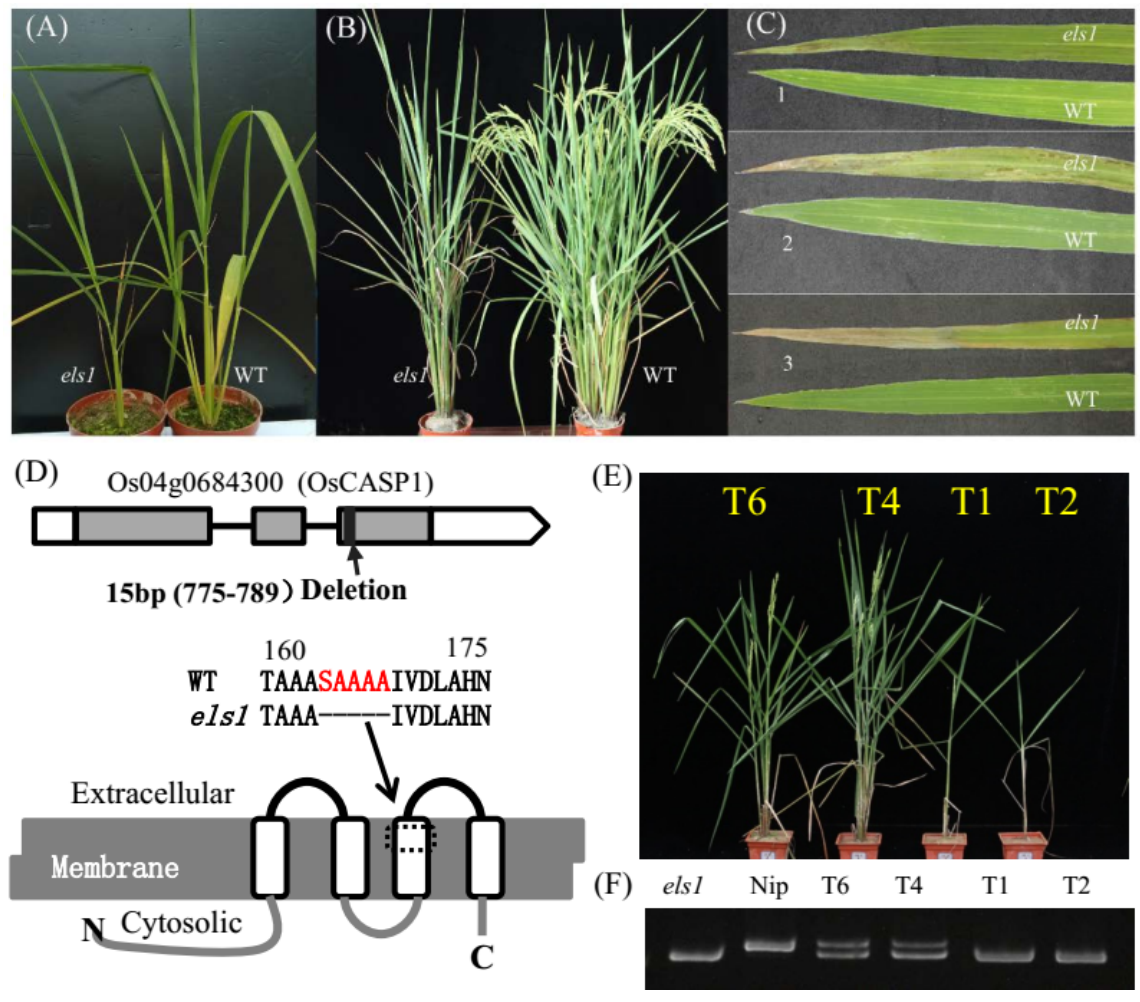

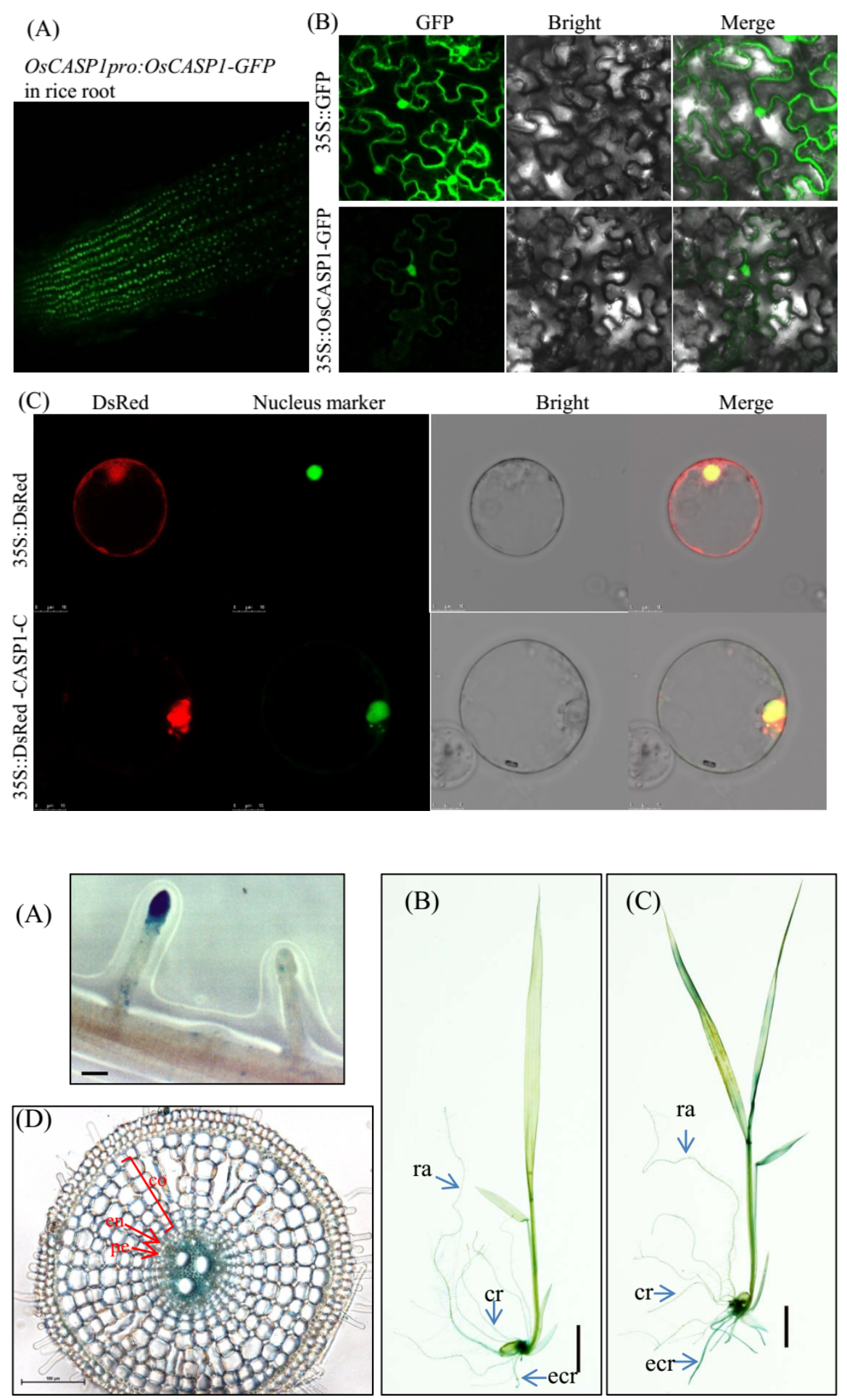
(A)

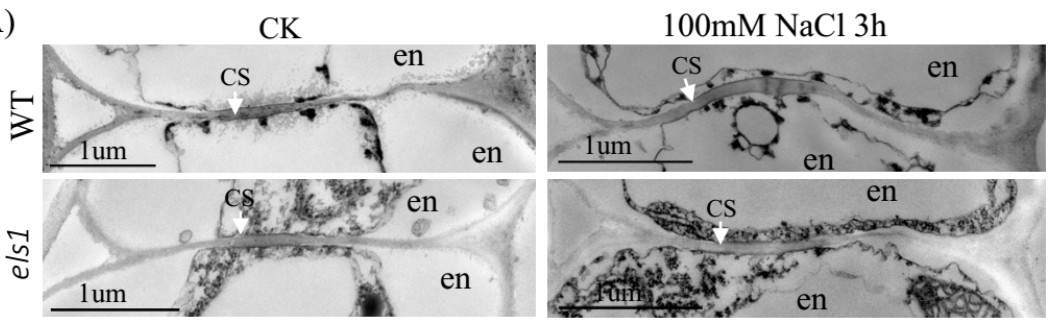

(B) Percentage of patchy suberization

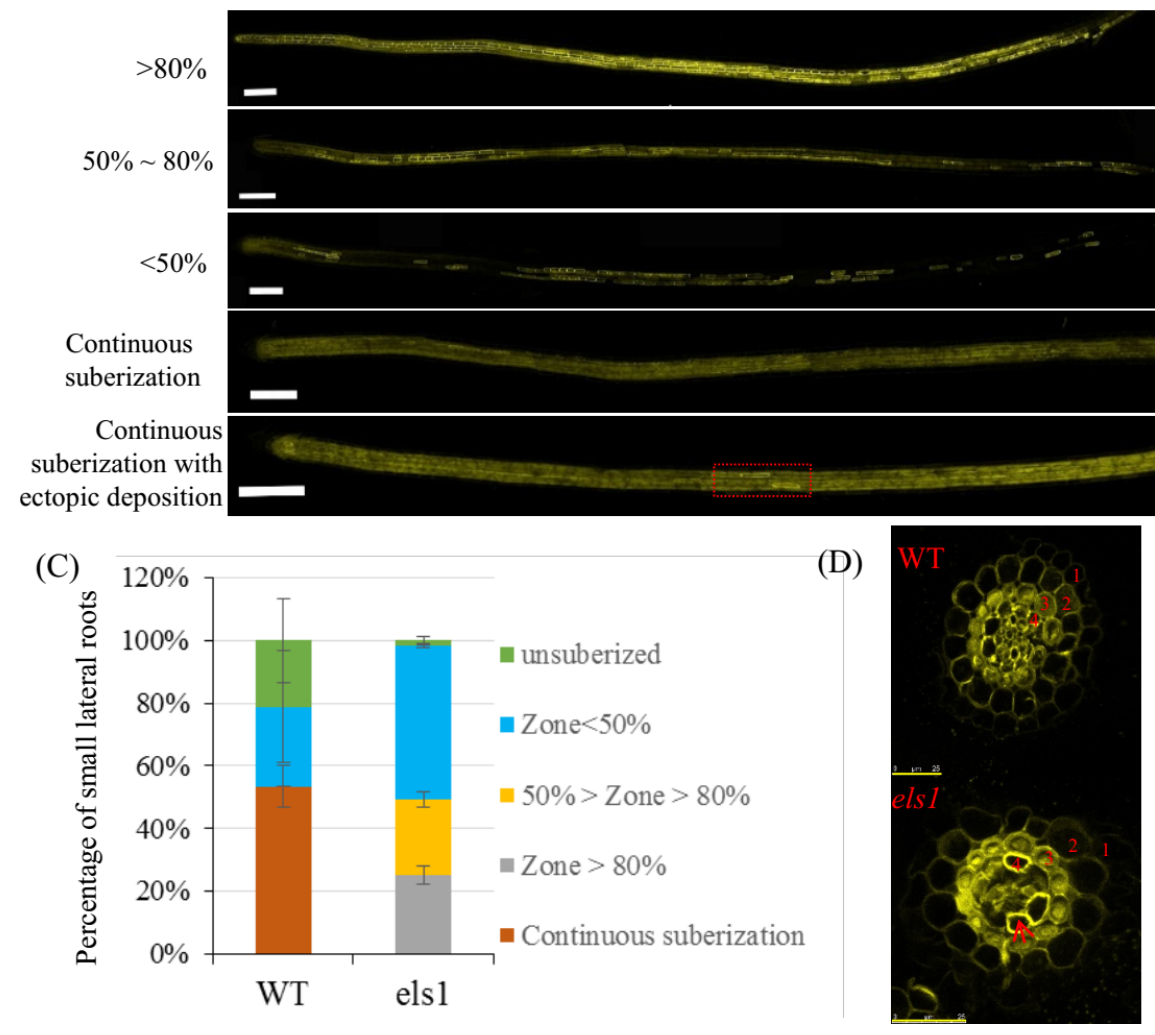




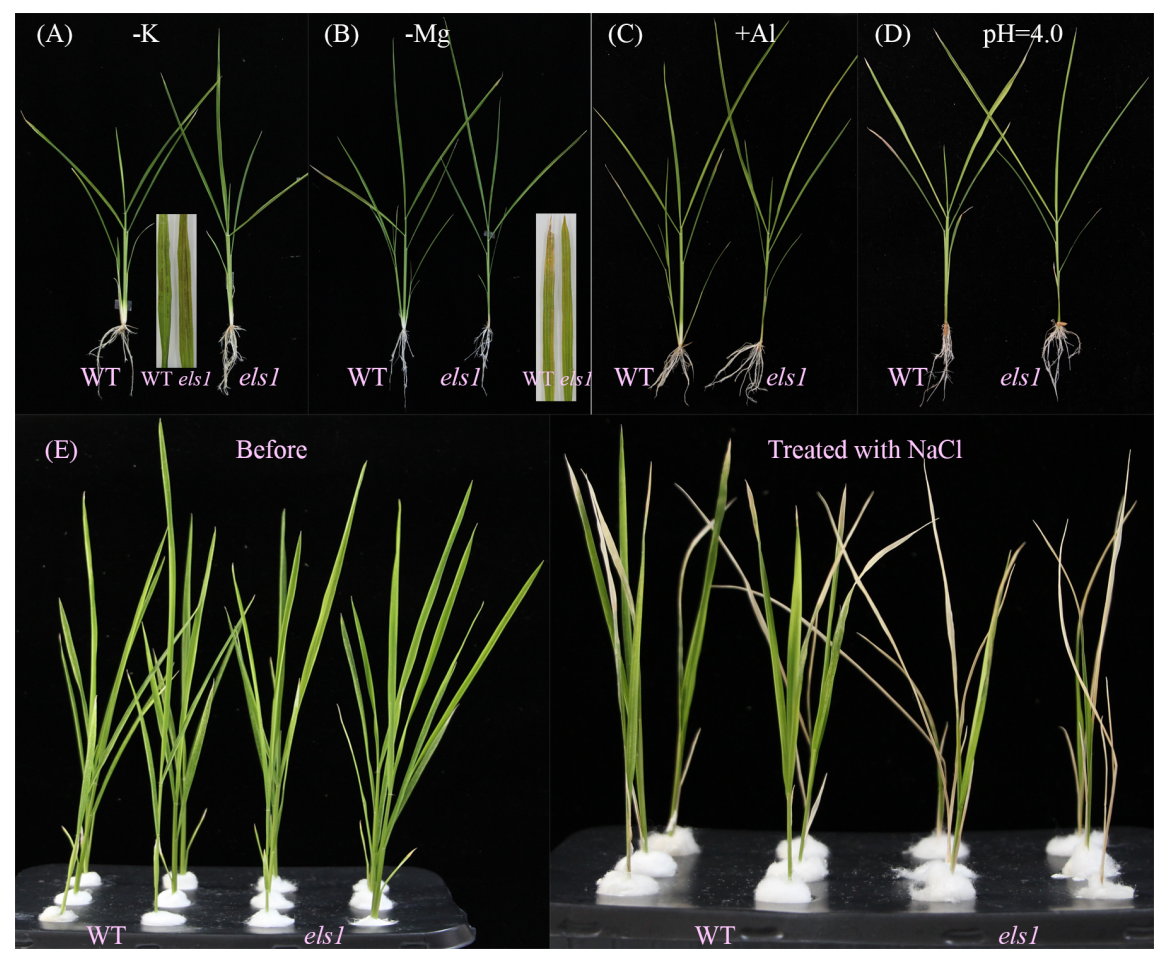



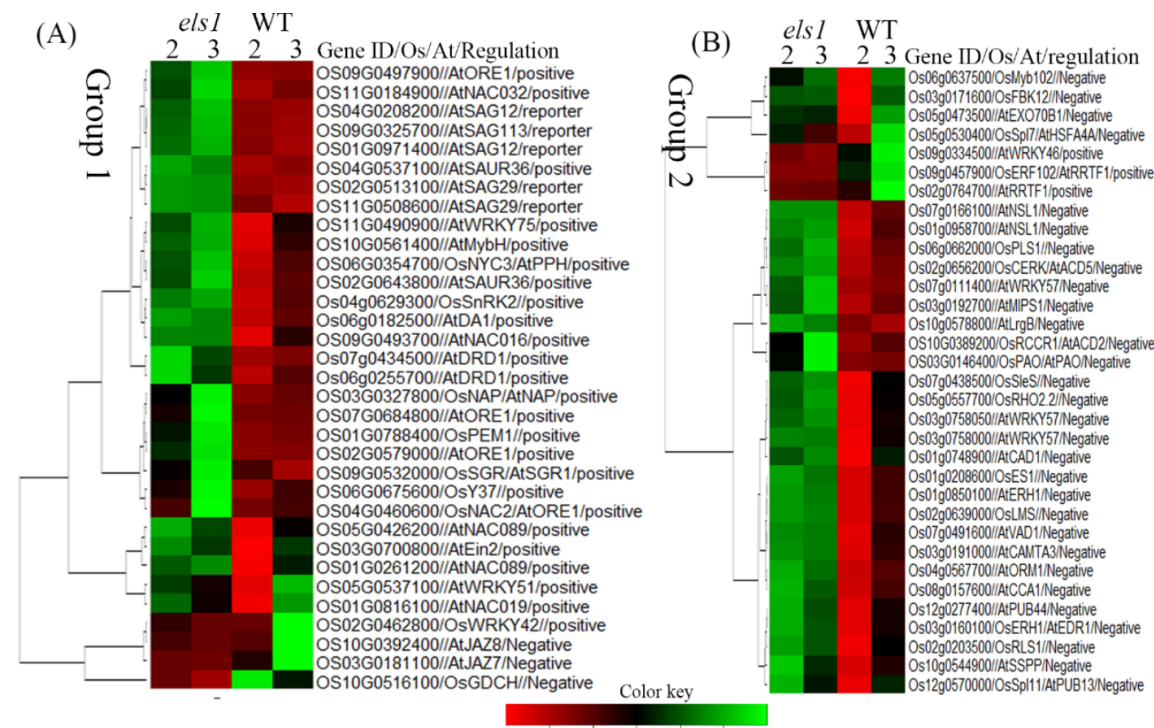

(C)

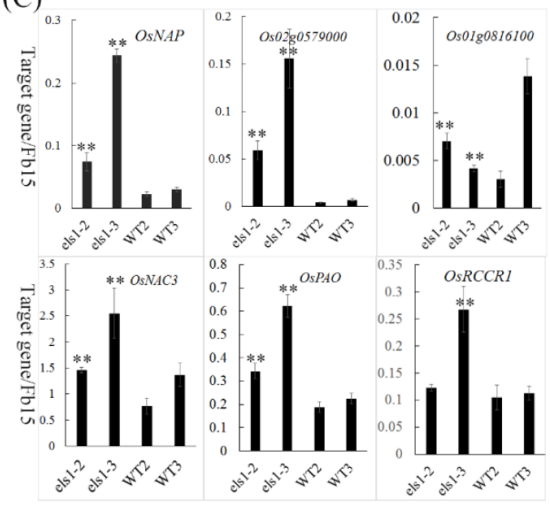

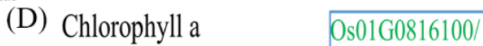

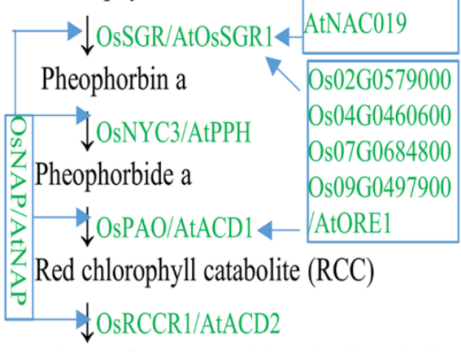

Primary fluorescent chlorophyll catabolite $\downarrow$

Chl degradation 
$O_{s} C A S P 1$ mutation

तु.

Ectopic suberin deposition in

small lateral roots

Nutrient starvation in plant

4

Nutrient remobilization

Fragile balance was destroyed

1

Chloroplast dismantle

Early leaf senescence 